\title{
Desenvolvimento e aplicação de ferramenta para remediação de lixões de resíduos sólidos urbanos
}

\section{Development and application of tool to assist in the remediation of municipal solid waste open dumps}

Data de entrada: $15 / 03 / 2019$

Data de aprovação: 15/04/2019

Juliano da Cunha Gomes ${ }^{1 *}$ | Ailton João Gonçalves Moreira² ${ }^{2}$ Alexandre Lioi Nascentes ${ }^{3}$ | Jose Julio Barrios Restrepo ${ }^{2}$ | Armando Borges de Castilhos Junior ${ }^{2}$

DOI: https://doi.org/10.36659/dae.2020.070

ORCID ID

Gomes JC (D) https://orcid.org/0000-0003-0312-4933

Moreira AJG (iD) https://orcid.org/0000-0003-1327-0096
Nascentes AL (D) https://orcid.org/0000-0002-3071-5969 Restrepo JJB (D) https://orcid.org/0000-0002-7575-6924

Castilhos Junior AB (D) https://orcid.org/0000-0003-0455-2585

\section{Resumo}

Lixões são locais onde os resíduos sólidos urbanos são descartados diretamente no solo, sem controle ambiental, causando impactos adversos ao meio ambiente e à saúde pública. $\mathrm{O}$ objetivo deste artigo foi desenvolver uma ferramenta auxiliar à remediação de lixões. A ferramenta, chamada de ReLix, foi testada por especialistas em dez lixões localizados no Brasil e na República de Cabo Verde. Oito lixões foram classificados como tendo impacto ambiental médio e dois lixões foram classificados como de alto impacto ambiental. 0 cenário de remediação predominante entre os lixões foi a remoção dos resíduos. O ReLix foi avaliado pelos especialistas como capaz de fornecer um diagnóstico apropriado, estabelecendo o nível de impacto e indicando as técnicas e o cenário de remediação com $92 \%$ de confiabilidade, permitindo às partes interessadas fazer análise caso a caso para garantir tomadas de decisão adequadas sobre as prioridades de remediação e a seleção do cenário de remediação de lixões.

Palavras-chave: Diagnóstico. Cenários de remediação. Resíduos sólidos urbanos. Lixões a céu aberto.

\section{Abstract}

Open dumps are places where municipal solid waste is disposed of directly in the ground, without environmental controls, causing adverse impacts on the environment and public health. The aim of this work was to develop a tool to assist in the remediation of municipal solid waste open dumps. The tool, called ReLix, was tested by experts at ten open dumps located in Brazil and in the Republic of Cabo Verde. Eight open dumps have a medium impact level and two have high impact level. The waste removal was the predominant remediation scenario. ReLix was evaluated by the experts as an appropriate diagnostic tool, establishing the level of impact and indicating the techniques and remediation scenario with $92 \%$ reliability, allowing the stakeholders to make a case-by-case analysis to ensure appropriate decision-making about the remediation priorities and in the selection of remediation scenarios of open dumps.

Keywords: Diagnosis. Remediation scenarios. Urban solid waste. Open dumps.

\footnotetext{
${ }^{1}$ Instituto Federal de Santa Catarina (IFSC) - Garopaba - Santa Catarina - Brasil.

${ }^{2}$ Universidade Federal de Santa Catarina (UFSC) - Florianópolis - Santa Catarina - Brasil.

${ }^{3}$ Universidade Federal Rural do Rio de Janeiro (UFRRJ) - Seropédica - Rio de Janeiro - Brasil.

* Autor correspondente: juliano.gomesaifsc.edu.br.
} 


\section{INTRODUÇÃO}

Os lixões são áreas onde os Resíduos Sólidos Urbanos (RSU) estão dispostos diretamente no solo sem o devido controle ambiental e seus principais efeitos são a degradação da qualidade do solo, ar, água superficial e subterrânea, proliferação de vetores de doenças e atração de pessoas com vulnerabilidade social que passam a "catar o lixo" como meio de sobrevivência (CASTILHOS JUNIOR et al., 2013; MONTEIRO et al., 2001).

Aproximadamente 50 a 100 milhões de toneladas de resíduos são depositados anualmente em lixões no mundo, predominantemente nos países em desenvolvimento. Muitos desses países não possuem dados representativos, e muitas vezes nenhuma informação referente à disposição final de seus RSU, fazendo com que as estimativas sejam precárias. Dados coletados em 87 países acerca da composição dos resíduos revelam que, do total anual gerado pelos países em desenvolvimento, de $54 \%$ a $64 \%$ são resíduos orgânicos, e a disposição final em lixões varia entre $13 \%$ e 33\% (HOORNWEG; BHADA-TATA, 2012).

Em 2016, o Brasil gerou cerca de 78,3 milhões de toneladas de RSU. Em média, 28\% dos municípios destinam seus RSU em lixões, 32\% em aterros controlados e $40 \%$ em aterros sanitários, ou seja, $60 \%$ dos municípios brasileiros ainda destinam seus resíduos em locais inadequados. Desde 2010, com a instituição da Política Nacional de Resíduos Sólidos no Brasil (PNRS), houve uma redução de apenas $1 \%$ no número dos municípios que depositam seus RSU em lixões (ABRELPE, 2013, 2015, 2017).

Embora a ocorrência de lixões no mundo seja antiga, nos países desenvolvidos eles são proibidos e já foram remediados há muitos anos, porém os países em desenvolvimento ainda estão em fase de implementação de suas políticas públicas sobre resíduos, como o Brasil e Cabo Verde, que enfrentam dificuldades para eliminar seus lixões. Essas informações apontam a necessidade de estratégias e/ou ferramentas que facilitem a eliminação dos lixões de RSU, sobretudo para municípios de pequeno porte, que constituem a maior parcela dos casos e tendem a ser desprovidos de corpo técnico especializado na gestão de RSU (BOYER et al., 1999; BURLAKOVS et al., 2017; DUBEY; CHAKRABARTI; PANDIT, 2016; JOSEPH et al., 2008; MAHESHI; STEVEN; KAREL, 2015; MARZOUGUI; BEN MAMMOU, 2006; MASI et al., 2014; USEPA, 1985, 1997; ZAÏRI et al., 2004; ZHOU et al., 2015).

Os principais métodos utilizados mundialmente para remediação de lixões são: a) o método presuntivo para remediação de aterros aplicado pela força aérea norte-americana (BOYER et al., 1999), b) o guia técnico francês de reabilitação de aterros (ADEME, 2005), c) o manual de reabilitação de lixões indiano (JOSEPH et al., 2008), d) o caderno técnico brasileiro para remediação de áreas degradadas por RSU (FEAM, 2010) e e) a ferramenta para diagnóstico ambiental de lixões (RAMOS et al., 2017).

O método presuntivo de remediação é um conjunto de medidas para a remediação dos aterros da força aérea norte-americana, os quais possuem características similares às de um lixão municipal no Brasil. O principal cenário de remediação que o método presuntivo usa é o confinamento dos resíduos após a escavação e tratamento das áreas mais contaminadas (hotspots) e a coleta e tratamento dos gases, água contaminada e lixiviados. Para isto estabelece uma série de fluxogramas para a escolha dos estudos a serem realizados na área e das técnicas remediações (BOYER et al., 1999).

No guia técnico para a reabilitação de aterros desenvolvido por Ademe (2005), o diagnóstico do lixão é feito em 4 etapas: 1) estudo da documentação e do histórico da área; 2) visita ao lixão; 3) Análise de risco e 4) escolha do cenário de 
remediação. Na terceira etapa é possível classificar os lixões em três categorias de risco: categoria $A$ (risco alto a médio), categoria $B$ (risco médio a baixo) e categoria $\mathrm{C}$ (risco baixo a nulo).

O manual de reabilitação de lixões indiano estabelece critérios para avaliação de perigos baseados no índice de risco numa escala de 0-1000. São cinco os potenciais de riscos: 1. Muito Alto (750-1000); 2. Alto (600-749); 3. Moderado (450-599); 4. Baixo (300-449); 5. Muito baixo (<300). Essa classificação norteia o processo decisório; nela, o risco muito alto aponta a necessidade de encerrar imediatamente as atividades do lixão e tomar medidas para mitigar os impactos. O risco muito alto determina o encerramento imediato do lixão e a mitigação dos impactos. 0 risco alto recomenda o encerramento imediato das atividades do lixão, porém a remediação é opcional. $\mathrm{O}$ risco moderado sugere a conversão imediata do lixão em aterro sanitário. 0 risco baixo preconiza que o lixão seja convertido em aterro sanitário gradualmente. Por fim, o risco muito baixo indica que o lixão tem potencial para ser um futuro aterro (JOSEPH et al., 2008).

O caderno técnico para remediação de áreas degradadas por RSU propõe cinco cenários de remediação: 1) remoção dos resíduos: remoção dos resíduos para um aterro sanitário; 2) remediação simples: encapsulamento ou confinamento dos resíduos por uma camada mínima de $50 \mathrm{~cm}$ de argila de baixa permeabilidade; 3) remediação parcial: a área sofre reconformação topográfica, os resíduos recebem uma cobertura vegetal com gramíneas, e um sistema de coleta e controle de água subterrânea, gases e lixiviados é implantado; 4) adequação provisória como aterro controlado: alternativa temporária para municípios com população inferior a 20.000 habitantes, que aguardam o licenciamento da área para conversão em aterro sanitário; 5) conversão em aterro sanitário: quando a área em que o lixão está localizado atende aos requisitos da ABNT NBR
13896/1997, possibilitando a operação de um aterro sanitário por um período superior a 15 anos (FEAM, 2010).

A ferramenta para diagnóstico de lixões foi desenvolvida pelo grupo do Laboratório de Pesquisa em Resíduos Sólidos (LARESO) da Universidade Federal de Santa Catarina (UFSC), Brasil. Trata-se de uma Ferramenta de Apoio à Decisão (FAD) para auxiliar o estabelecimento das prioridades de remediação entre um ou mais lixões de um mesmo município / região. A FAD fornece uma pontuação e um nível de impacto a cada lixão diagnosticado, listando-os por ordem decrescente de pontuação (RAMOS et al., 2017).

Existem dois grupos de pessoas envolvidas no desenvolvimento de FADs: as partes interessadas e os especialistas técnicos. As partes interessadas são aquelas que usam a FAD no processo de tomada de decisão, neste caso, os gestores municipais. Os especialistas técnicos são aqueles envolvidos no processo de construção, teste, avaliação e distribuição da FAD, geralmente no formato de programa de computador, aplicativos móveis ou web. Esses especialistas técnicos são cientistas, engenheiros de universidades ou do setor privado, de entidades governamentais ou de organizações não-governamentais (BARNHART et al., 2018).

Portanto, o objetivo deste artigo foi desenvolver e testar uma ferramenta, no formato de programa de computador, para auxiliar as partes interessadas na remediação de lixões de RSU em nível mundial.

\section{METODOLOGIA}

Este trabalho foi desenvolvido em duas etapas: 1) Desenvolvimento da ferramenta e 2) Aplicação da ferramenta. No desenvolvimento da ferramenta foram feitas a modelagem e a codificação dos módulos de diagnóstico de lixões e de 
indicação do cenário de remediação, no formato de programa de computador. Na aplicação da ferramenta foram realizados testes e avaliação por especialistas na área de resíduos sólidos.

\subsection{Desenvolvimento da ferramenta}

\subsubsection{Criação do módulo de diagnóstico}

A principal meta desse módulo é estabelecer as prioridades de remediação entre dois ou mais lixões. Por isso ele foi construído baseado no questionário de campo proposto inicialmente por Ramos et al. (2017) para diagnosticar lixões. Nesta perspectiva, foi proposto um sistema de pontua- ção para atribuir valores às situações que ocorrem no lixão e às respostas de cada pergunta. Esses valores somados constituem a pontuação do lixão, que é a principal fonte de informação que a ferramenta usa para estabelecer a prioridade de remediação entre dois ou mais lixões. 0 sistema de pontuação foi criado utilizando como referências a ponderação de atributos sugerida por Sánchez (2008), elementos do índice de risco proposto por Joseph et al. (2008), a hierarquização dos impactos ambientais estabelecidos pela Ademe (2005).

O questionário de campo está dividido em duas partes. Na primeira, é feita a identificação de quatro situações possíveis de ocorrer na área conforme a Fig. 1 .

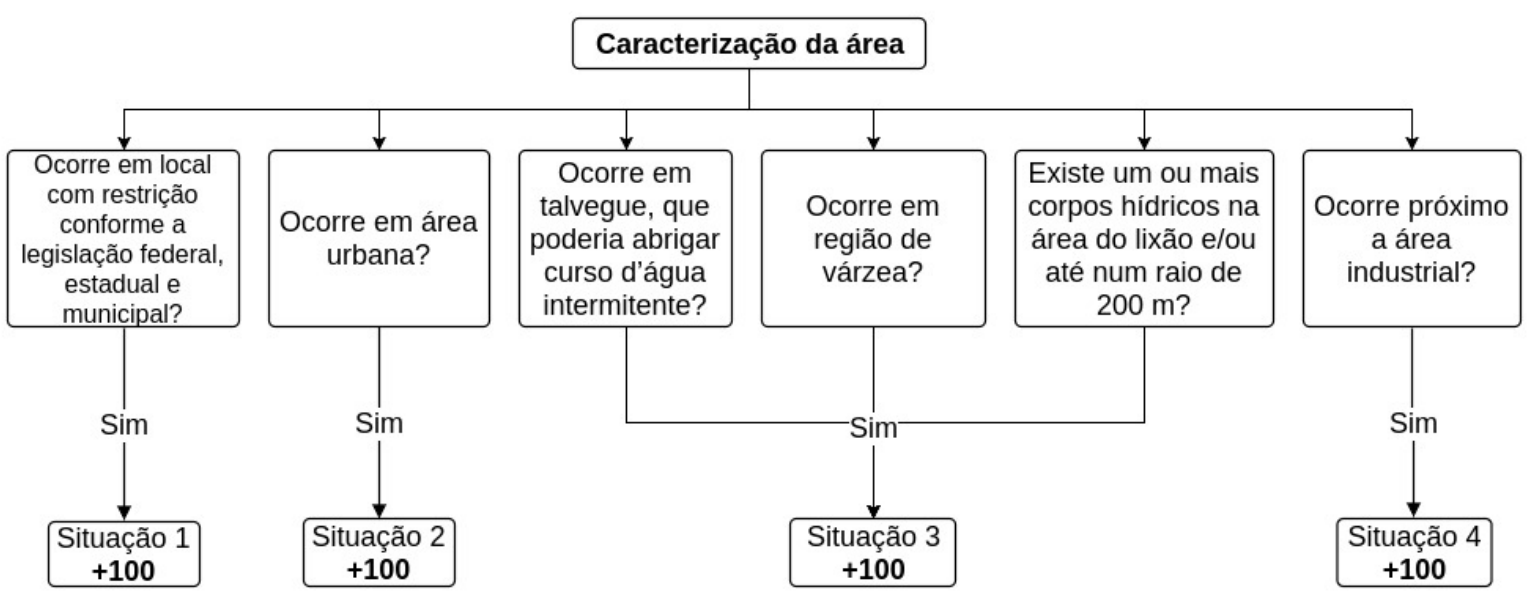

Figura 1: Situações possíveis de ocorrer em um lixão

Para cada situação foi atribuído um peso de 100 pontos, assim elas significam uma variação de zero a quatrocentos pontos na pontuação do lixão, em intervalos de cem pontos e o valor das situações é calculado conforme a Eq. 1.

$S=\sum_{S=0}^{4} 100$

onde $\mathrm{S}=$ valor das situações, e $\mathrm{S}=$ situações ocorridas.
$\mathrm{Na}$ segunda parte do questionário de campo existem sessenta e duas perguntas separadas por seis categorias: 1) Caracterização do lixão; 2) Solo e águas subterrâneas; 3) Águas superficiais; 4) Meio social; 5) Meio natural e paisagens; 6) Meio atmosférico.

Cada pergunta do questionário de campo foi estruturada de maneira que a primeira resposta é a que possui menor impacto, portanto menor valor, e a última resposta é a que pos- 
sui maior impacto, portanto maior valor. Como existe uma variação na quantidade de respostas de questão para questão, foi preciso normalizar os valores das respostas para tornar peso das questões equivalentes, utilizando a Eq. 2. Para calcular a pontuação do lixão foi elaborada a Eq. 3.

$$
\mathrm{Vr}=\sum_{\mathrm{r}=1}^{n} \frac{6}{n_{r}}
$$

onde $V_{r}=$ valor da resposta, $n_{r}=$ número de per guntas respondidas, $r=1$ (primeira resposta à pergunta) e $n=$ enésima resposta da pergunta.

$$
\mathrm{PL}=\mathrm{S}+\sum_{\mathrm{q}=1}^{n} V_{r}
$$

onde $\mathrm{PL}=$ pontuação do lixão, $\mathrm{S}=$ valor das situações, $v_{r}=$ valor da resposta, $r=1$ (primeira pergunta) e $n=$ enésima pergunta.

Para qualificar a pontuação atribuída pelo programa ao lixão, foi estabelecida a escala de impactos ambientais da Tabela 1, a qual possui um intervalo de 707 pontos que foi deduzido subtraindo-se a pontuação máxima e mínima que um lixão pode obter. Adotou-se o intervalo de $5 \%$ para o impacto reduzido, $15 \%$ entre o impacto reduzido e o impacto baixo, $30 \%$ entre o impacto baixo e o impacto médio, e $50 \%$ entre o impacto médio e o impacto alto.

Tabela 1: Escala de impactos ambientais proposta

\begin{tabular}{|c|c|c|}
\hline Nível de impacto & $\begin{array}{c}\text { Intervalo de } \\
\text { pontuação }\end{array}$ & $\begin{array}{c}\text { Diferença de } \\
\text { pontuação }\end{array}$ \\
\hline Reduzido & $>=125<=160$ & 35 pontos \\
\hline Baixo & $>=161<=266$ & 105 pontos \\
\hline Médio & $>=267<=479$ & 212 pontos \\
\hline Alto & $>=480<=832$ & 352 pontos \\
\hline
\end{tabular}

\subsubsection{Criação do módulo de cenários e técnicas de remediação}

Este módulo tem como objetivo indicar o cenário e as técnicas de remediação dos lixões diagnosticados pela ferramenta, sem considerar custos. Para isto, utilizaram-se como referências os cenários indicados por Feam (2010) e Ramos (2016), e técnicas de remediação apontadas por Bosmans et al.(2013), Boyer et al.(1999), Burlakovs et al. (2017), Dubey; Chakrabarti; Pandit, (2016), Gabbar; Aboughaly; Ayoub (2017), U.S EPA (1997, 2004, 2011) e Zaman (2010).

A revisão da literatura indicou a existência de três cenários de remediação: 1) Confinamento de resíduos; 2) Conversão para aterro sanitário e 3) Remoção de resíduos. As técnicas de remediação para esses cenários compreendem, de forma geral, técnicas de cobertura, técnica de remoção direta ou por mineração, de tratamento térmico, instalação de aterro sanitário, controle da área, controle da água subterrânea, coleta e tratamento de lixiviado e tratamento de gases.

Assim como na etapa de diagnóstico, foi proposto um sistema de pontuação onde a técnica de remediação é obtida em função do número de critérios satisfeitos no momento de preenchimento do questionário de campo, conforme a Eq. 4. Devido a um máximo de cinco critérios por técnica, o valor pode variar de zero a cinco, onde zero indica que nenhum critério foi escolhido e, portanto, não há necessidade de usar a técnica. Quanto maior a pontuação da técnica, maior sua relevância e prioridade de aplicação ao cenário.

$$
\mathrm{Vt}=\sum_{\mathrm{c}=1}^{n} \frac{5}{n_{c}}
$$

onde $V_{t}=$ pontuação da técnica, $c=1$ (1 critério selecionado), $n$ = enésimo critério selecionado e $n_{c}=$ número de critérios selecionados. 
Da mesma forma, a pontuação dos cenários de remediação foi extraída da quantidade de técnicas selecionadas, de acordo com a Eq. 5. Esse valor também pode variar de zero a cinco, pois há, no máximo, cinco técnicas por cenário. Quanto maior a pontuação do cenário, maior a sua aplicabilidade.

$\mathrm{Vc}=\sum_{\mathrm{t}=1}^{n} \frac{5}{n_{t}}$

onde $V_{c}=$ pontuação do cenário, $t=1$ (1 técnica selecionada), $\mathrm{n}$ = enésima técnica selecionada e $n_{t}=$ número de técnicas selecionadas.

Desta forma, a ferramenta permite que todos os cenários de pontuação sejam listados, bem como suas respectivas técnicas de correção. 0 cenário que obtém a pontuação mais alta é o mais indicado.

Levando em consideração aspectos legais e características relevantes na área de um lixão, uma avaliação qualitativa foi adicionada após o estabelecimento das pontuações do cenário. Para isso, a ferramenta avalia a propensão para um determinado cenário de acordo com o fluxograma apresentado na Fig. 2 e adiciona seis pontos ao escore do cenário, obtendo assim a pontuação total do cenário, conforme a Eq. 6 . Esses seis pontos extras foram definidos utilizando o valor máximo possível em um cenário (cinco) mais um, para não haver possibilidade de empate.

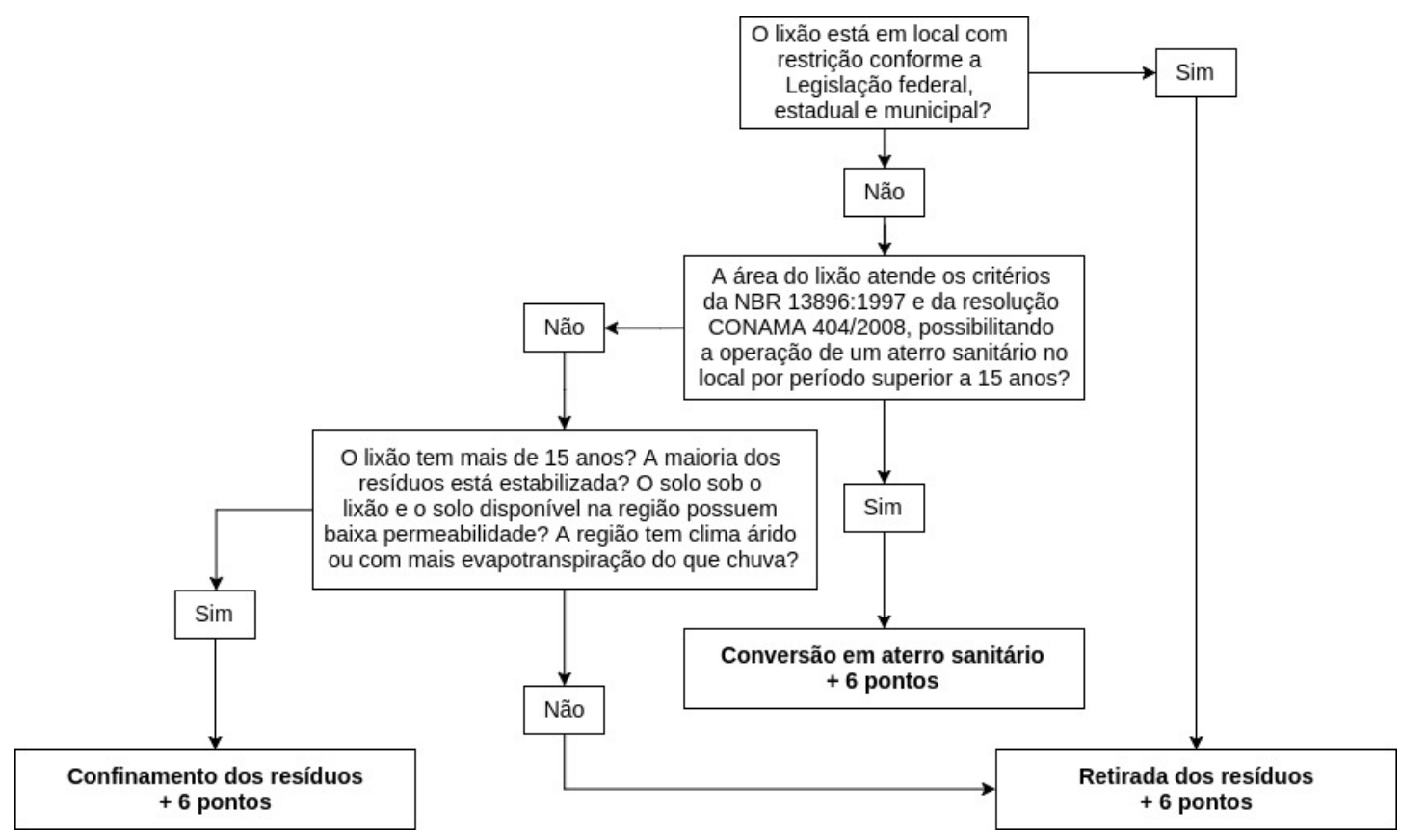

Figura 2: Fluxograma de atribuição de pontuação extra ao cenário de remediação

$\mathrm{Vt}_{c}=\mathrm{Vc}+6$

onde $V t_{c}=$ valor total do cenário, e $V_{c}=$ valor do cenário.

\subsubsection{Codificação do programa de computador}

A ferramenta foi codificada utilizando-se a linguagem de programação $\mathrm{C}++$ com o ambien- 
te de desenvolvimento integrado Qt Creator e sistema de gerenciamento de banco de dados SQLite, pois ambos são multiplataforma e de código fonte aberto, com documentação de fácil acesso e ampla comunidade de desenvolvedores. A base de dados do programa foi modelada através de diagramas de classe de associação binária da Linguagem de Modelagem Unificada (UML), conforme a Fig. 3. O programa foi nomeado como ReLix em referência ao termo Remediação de Lixões e encontra-se disponível no repositório do desenvolvedor ${ }^{1}$.

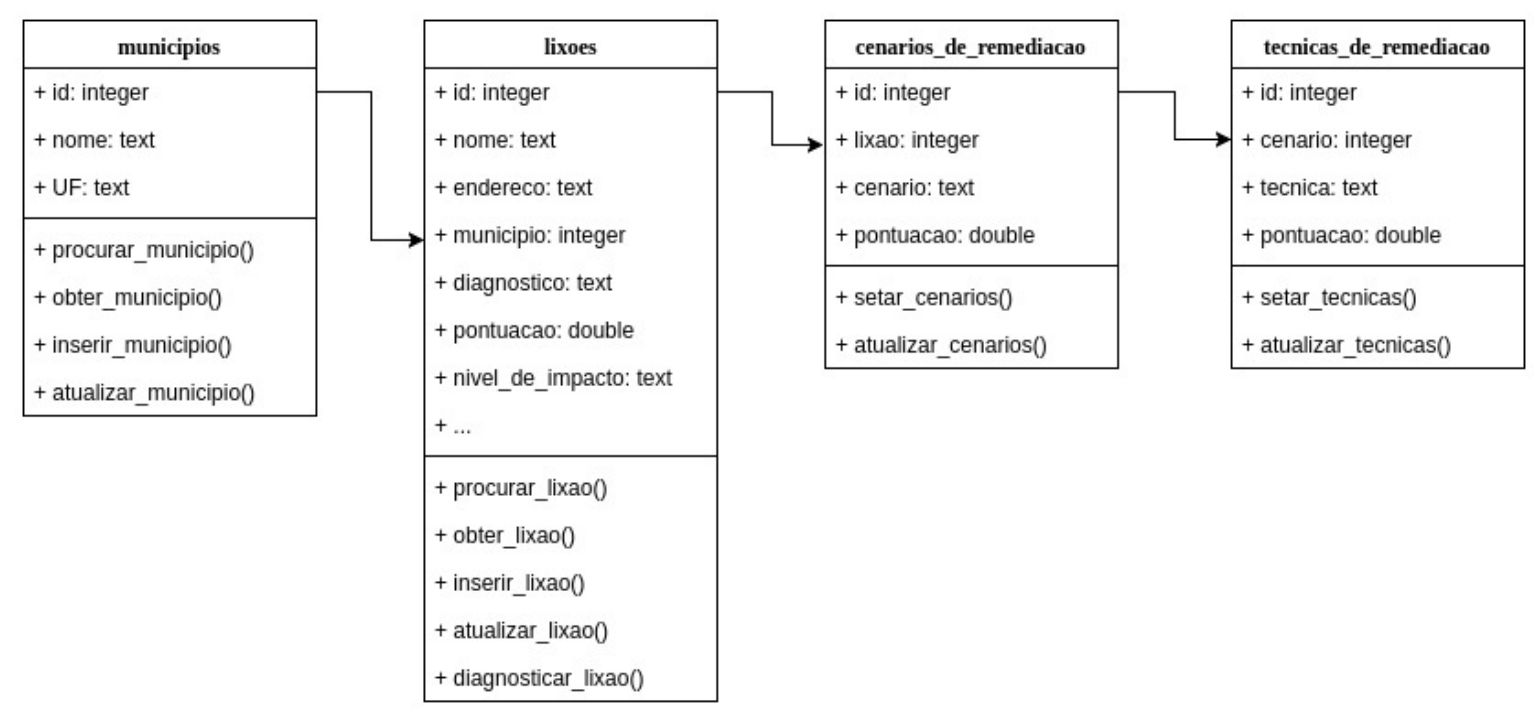

Figura 3 - Modelo da base de dados do ReLix

\subsection{Aplicação e avaliação do programa de computador}

O ReLix foi aplicado em dez lixões, seis deles estavam situados em diferentes estados brasileiros e quatro estavam na República de Cabo Verde. A seleção desses lixões se deu em função da existência prévia de dados sobre as áreas e da disponibilidade de especialistas com experiência na área de resíduos sólidos para testar e avaliar o ReLix.

A avaliação do ReLix nesta etapa foi feita por meio de uma banca com quatro especialistas, dois deles brasileiros e dois de Cabo Verde. $\mathrm{Na}$ ocasião, os especialistas receberam as seguintes instruções: ler o manual, instalar o programa, cadastrar as informações dos lixões no progra- ma, executar o diagnóstico e por fim preencher o questionário de avaliação do ReLix.

O método de avaliação utilizado no questionário de avaliação do ReLix foi a escala Likert de cinco pontos, pois ela possibilita ao entrevistado manifestar sua resposta de forma mais fácil, rápida e precisa, por possuir maior variedade de respostas quando comparada ao questionário tradicional contendo apenas o sim e não como opções de resposta.

Por isso, durante a elaboração do questionário, seguiram-se as recomendações de Dalmoro; Vieira (2014) no sentido de padronizar o formato das respostas buscando sempre um ponto neutro como opção da resposta número três, elaborar um número razoável de questões para

${ }^{1}$ https://github.com/jcgomes/ReLix 
não sobrecarregar o avaliador e utilizar as âncoras verbais somente nas extremidades da escala para não poluir visualmente a mesma.
As perguntas do questionário de avaliação do ReLix que usam a escala likert como resposta estão listadas na Tabela 2.

Tabela 2: Perguntas do questionário de avaliação do ReLix

- O manual do usuário é de fácil entendimento e permite aprender a utilizar o programa sem auxílio técnico?
- A interface do programa é amigável e de fácil utilização?
- Com base na sua experiência, o diagnóstico feito pelo programa é coerente com o seu diagnóstico?
- O diagnóstico feito pelo programa corresponde ao diagnóstico do caso real?
- O programa é capaz de apoiar a decisão sobre as prioridades de remediação entre dois ou mais lixões?
- Tendo em vista que o programa classifica os cenários de remediação para determinado lixão em ordem decrescente de
pontuação e que o cenário de maior pontuação é o mais indicado, a ordem dos cenários estabelecida pelo programa
está na sequência que você entende como adequada?
- O cenário indicado pelo programa equivale ao do cenário real ou ao cenário que você entende como adequado?
- Quão próximos dos motivos reais estão os motivos fornecidos pelo programa para a indicação do cenário (destacado em
amarelo no rodapé da aba cenário)?
- As técnicas de remediação indicadas pelo programa são equivalentes às técnicas aplicadas no caso real ou as que você
entende como adequadas para a remediação do lixão?

\section{RESULTADOS E DISCUSSÃO}

\subsection{Apresentação do programa desenvolvido}

$\mathrm{Na}$ tela de consulta de lixões, por padrão, todos os lixões são listados em ordem decrescente de pontuação, conforme é possível constatar na
Fig. 4, bastando filtrar o município / região. Essa funcionalidade facilitou a visualização de todos os lixões cadastrados no banco de dados, permitiu a aplicação de filtros e a ordenação de dados de forma simplificada, auxiliando o estabelecimento das prioridades de remediação.

\begin{tabular}{|c|c|c|c|c|c|}
\hline ID & Nome do lixāo & Município / regiāo & Pontuaçāo ^ & \multicolumn{2}{|c|}{ Nivel de impacto } \\
\hline 9 & Lixāo CV4 & Cabo Verde & 709 & \multicolumn{2}{|l|}{ Alto } \\
\hline 4 & Lixāo RS & Porto Alegre & 538 & \multicolumn{2}{|l|}{ Alto } \\
\hline 7 & Lixāo SC2 & Içara & 476 & \multicolumn{2}{|l|}{ Médio } \\
\hline 8 & Lixāo SC3 & Florianópolis & 464 & \multicolumn{2}{|l|}{ Médio } \\
\hline 5 & Lixāo SP & Sāo Carlos & 427 & \multicolumn{2}{|l|}{ Médio } \\
\hline 1 & Lixāo CV3 & Cabo Verde & 394 & \multicolumn{2}{|l|}{ Médio } \\
\hline 2 & Lixāo CV1 & Cabo Verde & 373 & \multicolumn{2}{|l|}{ Médio } \\
\hline 6 & Lixāo SC1 & Sāo Bento do Sul & 324 & \multicolumn{2}{|l|}{ Médio } \\
\hline 11 & Lixāo RJ & Vassouras & 320 & \multicolumn{2}{|l|}{ Médio } \\
\hline 3 & Lixāo CV2 & Cabo Verde & 279 & \multicolumn{2}{|l|}{ Médio } \\
\hline \multicolumn{2}{|c|}{ Campo: } & \multicolumn{4}{|l|}{ Parmâmetro: } \\
\hline \multicolumn{2}{|c|}{ Município / regiāo - } & & & * Adicionar & (C) S- \\
\hline
\end{tabular}

Figura 4: Tela de consulta de lixões do programa ReLix 
A tela principal do ReLix, ilustrada na Fig. 5, é onde acontece o cadastro, diagnóstico e indicação do cenário e técnicas de remediação de um lixão. Ela foi dividida em dez abas localizadas na parte superior da tela. As sete primeiras referem-se as categorias do questionário de campo e todos os seus campos são de preenchimento obrigatório. É possível reconhecer facilmente a aba que está sendo preenchida, pois ela fica destacada na cor azul. Para a maioria das opções de resposta nesta tela, foram utilizados objetos do tipo botão de rádio ou de checagem, para que cada resposta fosse dada com apenas um clique. $\mathrm{Na}$ oitava aba pode-se inserir fotos do lixão. Esta aba é de preenchimento opcional.

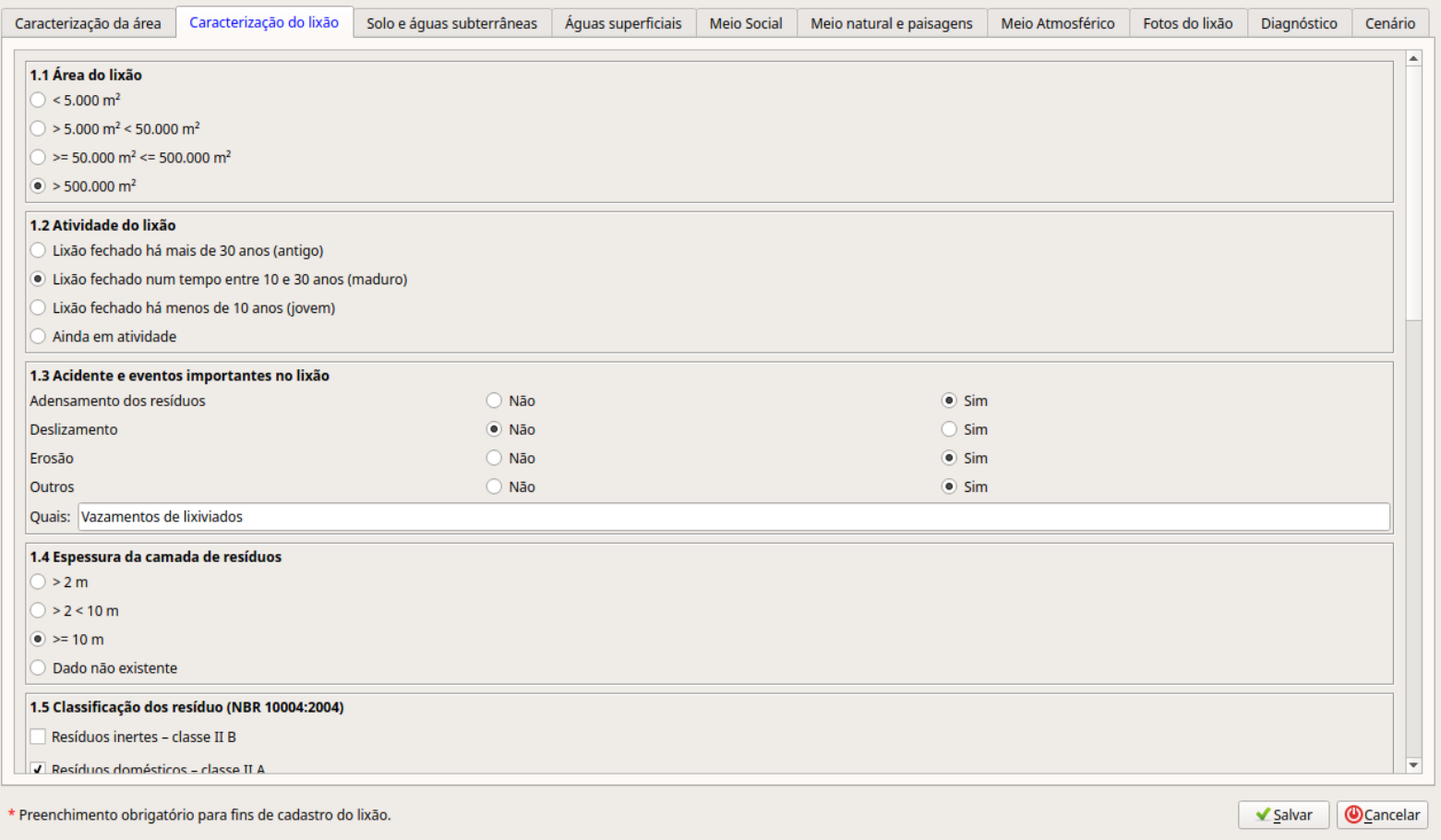

Figura 5: Tela de cadastro, diagnóstico e indicação do cenário e técnicas de remediação do ReLix

Após preencher as sete primeiras abas e salvar as informações, o ReLix preenche automaticamente as duas últimas abas, referentes ao diagnóstico e a indicação do cenário e técnicas de remediação, visto nas Figs. 6 e 7. Organizar todas as categorias de informações de um lixão em uma tela dividida por abas facilitou a operacionalização do programa, pois o usuário consegue encontrar as informações de forma rápida e direta. 


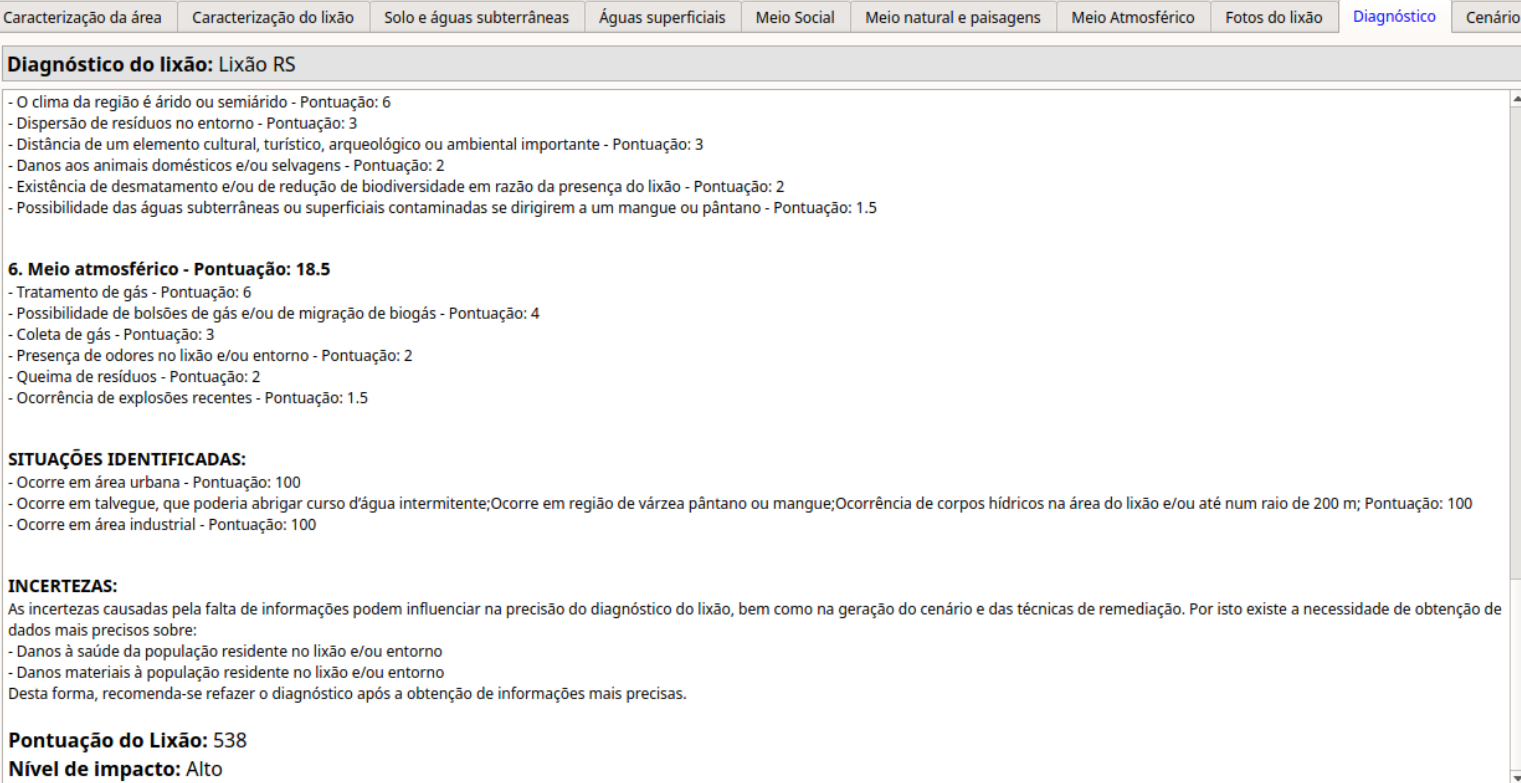

Figura 6: Tela de um diagnóstico de lixão feito pelo ReLix

\begin{tabular}{|c|c|c|c|c|c|c|c|c|c|}
\hline Caracterizaçāo da área & Caracterização do lixāo & Solo e águas subterrâneas & Águas superficiais & Meio Social & Meio natural e paisagens & Meio Atmosférico & Fotos do lixāo & Diagnóstico & Cenário \\
\hline \multicolumn{10}{|c|}{ Cenário(s) de remediaçāo para o lixāo: Lixão RS } \\
\hline \multicolumn{9}{|c|}{ Cenário } & Pontuação \\
\hline \multicolumn{9}{|l|}{ Retirada dos residuos } & 8,5 \\
\hline \multicolumn{9}{|c|}{ Confinamento dos resíduos } & 4 \\
\hline \multicolumn{9}{|c|}{ Conversāo em aterro sanitário } & 3,3334 \\
\hline \multicolumn{10}{|c|}{ Técnica(s) de remediação indicada(s) para o cenário selecionado: } \\
\hline \multicolumn{8}{|c|}{ Técnica } & \multicolumn{2}{|c|}{ Pontuação } \\
\hline \multicolumn{8}{|c|}{ Remoçāo dos resíduos por mineraçāo com tratamento térmico por gaseificaçāo } & \multicolumn{2}{|c|}{5} \\
\hline \multicolumn{8}{|c|}{ Coleta de lixiviado / água contaminada através do sistema de bombeamento pressurizado } & \multicolumn{2}{|l|}{5} \\
\hline \multicolumn{8}{|c|}{ Controle da água subterrânea com poços de extração e drenagens de subsuperficie com paredes de suspensão degradáveis ou paredes de tratamento } & \multicolumn{2}{|l|}{5} \\
\hline \multicolumn{8}{|c|}{ Sistema ativo de coleta de gases } & \multicolumn{2}{|l|}{5} \\
\hline \multicolumn{8}{|c|}{ Tratamento fisico, químico ou biológico da água contaminada / lixiviado } & \multicolumn{2}{|l|}{5} \\
\hline \multicolumn{8}{|c|}{ Ventilação passiva dos gases } & \multicolumn{2}{|l|}{5} \\
\hline \multicolumn{8}{|l|}{ Controle da área } & \multicolumn{2}{|l|}{2,5} \\
\hline
\end{tabular}

Valor total do cenário: R\$ 0,00

Cenário indicado: Retirada dos resíduos, porque embora o lixão não esteja em local com restriçāo conforme a legislaçāo, a área do lixāo não atende os critérios da NBR 13896:1997 e da resoluçāo CONAMA 404/2008, nāo possibilitando a operaçāo de um aterro sanitário no local por período superior a 15 anos; A maioria dos resíduos nāo estāo estabilizados; O clima da regiāo nāo é árido ou semiárido; A regiāo nāo tem mais evapotranspiração do que chuvas:

Figura 7: Tela de indicação de cenário e técnicas de remediação feito pelo ReLix 


\subsection{Aplicação e avaliação do programa de computador}

O ReLix foi aplicado em dez lixões, seis em diferentes estados brasileiros, Santa Catarina (SC1, SC2 e SC3), São Paulo (SP) e Rio de Janeiro (RJ), Rio Grande do Sul (RS) e quatro na república de Cabo Verde (CV1, CV2, CV3 e CV4). A Tabela 3 fornece um resumo das pontuações obtidas na aplicação do programa nos lixões, os quais estão distribuídos na tabela por ordem crescente de pontuação e consequentemente de impacto ambiental.

Tabela 3: Resumo dos resultados da aplicação do ReLix nos 10 lixões

\begin{tabular}{|c|c|c|c|c|c|c|c|c|c|c|}
\hline \multirow{2}{*}{ Parâmetro } & \multicolumn{10}{|c|}{ Lixão } \\
\hline & CV2 & RJ & SC1 & CV1 & CV3 & SP & SC3 & SC2 & RS & CV4 \\
\hline $\begin{array}{c}\text { Caracterização do } \\
\text { lixão }\end{array}$ & 74 & 61,5 & 68 & 81,5 & 73,5 & 82,5 & 64 & 67 & 69 & 81 \\
\hline $\begin{array}{l}\text { Solos eáguas } \\
\text { subterrâneas }\end{array}$ & 55 & 56 & 49 & 50 & 53 & 68,5 & 66,5 & 62,5 & 51 & 65,5 \\
\hline Águas superficiais & 24,5 & 24,1 & 29,1 & 20,5 & 24,5 & 33,5 & 32 & 31,5 & 25,5 & 27 \\
\hline Meio social & 57 & 33,5 & 31 & 57,5 & 70 & 66 & 42 & 40 & 44 & 60 \\
\hline $\begin{array}{l}\text { Meio natural e } \\
\text { paisagens }\end{array}$ & 33,5 & 28 & 28 & 35,5 & 38,5 & 43,5 & 34,5 & 48 & 29,5 & 40,5 \\
\hline Meio Atmosférico & 34,5 & 16,5 & 18,5 & 31 & 34,5 & 32,5 & 24,5 & 26,5 & 18,5 & 34,5 \\
\hline Situações ocorridas & 0 & 100 & 100 & 100 & 100 & 100 & 200 & 200 & 300 & 400 \\
\hline Pontuação do lixão & 279 & 320 & 324 & 376 & 394 & 427 & 464 & 476 & 538 & 709 \\
\hline Nível de impacto & $M$ & $M$ & $M$ & $M$ & $M$ & $M$ & $M$ & $M$ & A & A \\
\hline Cenário indicado & RR & RR & $\mathrm{CA}$ & RR & CR & RR & RR & RR & RR & RR \\
\hline
\end{tabular}

Legenda: $M$ = Médio; $A$ = Alto; $R R=$ Remoção de resíduos; $C A$ = Conversão em aterro sanitário. $C R=$ Confinamento de resíduos

Na Fig. 8 é possível observar em que categoria os lixões foram mais impactantes de acordo com o diagnóstico feito pelo ReLix, sem considerar as situações identificadas nas áreas. $\mathrm{Na}$ categoria caracterização do lixão foram os lixões SP, CV1 e CV4 devido ao seu porte, atividades, acidentes, tipos de resíduos recebidos e falta de impermeabilização superior. $\mathrm{Na}$ categoria solo e águas subterrâneas os lixões SP, SC3, CV4 e SC2 foram mais impactantes pela falta de impermeabilização inferior, falta de coleta e tratamento dos lixiviados e contaminação da água subterrânea. $\mathrm{Na}$ categoria meio social os lixões mais impactantes foram os CV3, SP e CV4 em função da constata- ção de atividades nas proximidades, presença de animais e catadores. Na categoria meio natural e paisagens os lixões SC2, SP e CV4 foram mais impactantes em decorrência da dispersão de resíduos no entorno e pela falta de informações. $\mathrm{Na}$ categoria meio atmosférico os lixões mais impactantes foram os CV2, CV3 e CV4 devido à presença de odores, queima de resíduos, falta de coleta e tratamento dos gases. De maneira geral, os lixões SP e CV4 estiveram presentes entre os mais impactantes em todas as categorias, seguidos dos lixões SC2 e CV3, presentes entre os mais impactados em duas categorias. 


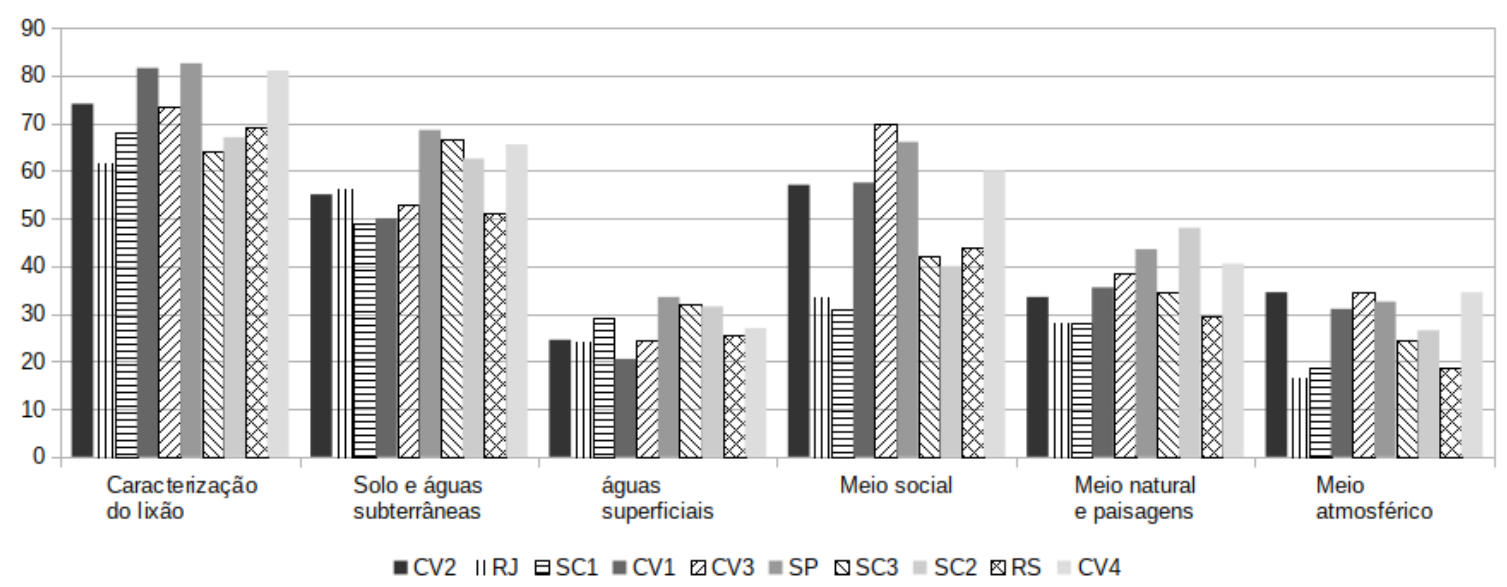

Figura 8: Pontuação dos lixões por categoria

Contudo, as situações ocorridas nas áreas dos lixões, identificadas pelo ReLix, foram determinantes no estabelecimento dos níveis de impacto de cada lixão, sobretudo para os lixões CV4 e RS, que foram classificados como de alto impacto ambiental. No lixão CV4 ocorreram as quatro situações possíveis, enquanto no lixão RS ocorreram três, conforme observado na Tabela 3.

O cenário indicado pelo ReLix para os lixões CV2, RJ, CV1, SP, SC3, SC2 e RS foi a retirada dos resíduos. Entre os aspectos legais e características relevantes na definição do cenário, levou-se em consideração que os lixões não atendem aos critérios legais e técnicos para a conversão do lixão em aterro sanitário, a maioria dos resíduos não está estabilizada, o solo sob o lixão e o solo disponível na região não possuem baixa permeabilidade, o clima da região não é árido ou semiárido e a região não tem mais evapotranspiração do que chuvas.

Para o lixão SC1 o cenário foi a conversão em aterro sanitário. Dos aspectos legais e características relevantes na definição do cenário, observou-se que o lixão não está em local com restrição conforme a legislação, e a área do lixão atende aos critérios legais e técnicos para a conversão do lixão em aterro sanitário, possibilitando sua operação no local por período superior a 15 anos.
Para o lixão CV3 o ReLix indicou o cenário de confinamento dos resíduos, pois constatou-se que o lixão não está em local com restrição conforme a legislação, a área do lixão não atende aos critérios legais e técnicos para a conversão do lixão em aterro sanitário, não possibilitando a operação de um aterro sanitário no local por período superior a 15 anos, porém o lixão tem mais de 30 anos de idade e por isso pressupõe-se que a geração de gases e a lixiviação dos resíduos restantes é relativamente baixa. A maioria dos resíduos está estabilizada, o solo sob o lixão e o solo disponível na região possuem baixa permeabilidade e a região tem clima árido ou com mais evapotranspiração do que chuvas.

No caso do lixão CV4, o cenário da retirada dos resíduos foi indicado porque o lixão está em local com restrição conforme a legislação.

Conforme observado na Tabela 3, dos quatro lixões de Cabo Verde, os três situados na llha de Santiago (CV1, CV2 e CV3) estavam entre os cinco que apresentaram menor pontuação, seguidos dos lixões brasileiros de Santa Catarina, São Paulo, Rio Grande do Sul e finalmente o lixão de Cabo Verde, localizado na Ilha do Fogo (CV4).

Ainda que a maioria dos lixões diagnosticados pelo programa tenha sido classificada como de médio impacto, eles possuem faixas de valores 
distintas, que os dividem em três grupos. No primeiro grupo estão os três lixões de Cabo Verde, que obtiveram pontuação entre 200 e 300 pontos. No segundo grupo incluem-se os três lixões de Santa Catarina, com pontuação entre 300 e 400 pontos. 0 terceiro grupo é composto pelos lixões de SP, RS e CV4, que tiveram pontuação acima de 400 pontos, sendo que os lixões RS e CV4 foram classificados com nível de alto impacto, especialmente pela identificação de duas situações para o lixão RS e três para o lixão CV4.

Em resumo, o lixão CV4 tem maior prioridade de remediação, seguido do lixão RS, SP, SC2, SC3, $\mathrm{SC} 1, \mathrm{CV} 3, \mathrm{CV} 2$ e CV1. Essa prioridade é sugerida automaticamente pelo ReLix ao listar todos os lixões em ordem decrescente de pontuação, conforme visto na Tabela 4. Essa funcionalidade facilita a interpretação do diagnóstico e o processo decisório.

É preciso destacar que, dentre os lixões classificados com nível de impacto médio, o lixão que obteve menor pontuação (Lixão CV1) estava a 30 pontos do nível de impacto baixo, enquanto o que obteve maior pontuação (Lixão SP) estava a 9 pontos do nível de impacto alto, portanto, embora eles tenham sido classificados como impacto médio, ambos estavam próximos aos limites de mudança de nível de impacto. Isso permite que o gestor analise caso a caso para tomar a decisão mais adequada sobre as prioridades de remediação e a escolha do cenário e técnicas de remediação entre dois ou mais lixões.

Quanto aos resultados da avaliação do ReLix observados na Tabela 4, a média geral da avaliação feita pelos especialistas foi 4,6. Tendo em vista que a pontuação máxima é 5 pontos, pode-se afirmar que, numa escala de confiabilidade de 0 a $100 \%$, o programa obteve $92 \%$ de confiabilidade para os lixões avaliados, o que significa que foi consenso entre os especialistas que o ReLix é fácil de entender e usar, que o diagnóstico feito pelo programa é coerente com o diagnóstico feito pelos especialistas, que o programa é capaz de auxiliar adequadamente as partes interessadas no estabelecimento das prioridades de remediação e na indicação do cenário e técnicas de remediação.

Tabela 4: Resumo da avaliação do ReLix feita pelos especialistas

\begin{tabular}{|c|c|c|c|c|c|}
\hline Questão & E1 & E2 & E3 & E4 & Média \\
\hline $\begin{array}{l}\text { O manual do usuário é de fácil entendimento e permite aprender a utilizar o programa } \\
\text { sem auxílio técnico? }\end{array}$ & 5 & 5 & 5 & 5 & 5,0 \\
\hline A interface do programa é amigável e de fácil utilização? & 4 & 5 & 5 & 5 & 4,8 \\
\hline $\begin{array}{l}\text { Com base na sua experiência, o diagnóstico feito pelo programa é coerente com o seu } \\
\text { diagnóstico? }\end{array}$ & 4 & 5 & 5 & 5 & 4,8 \\
\hline O diagnóstico feito pelo programa, corresponde ao diagnóstico do caso real? & 1 & 5 & 5 & 5 & 4,0 \\
\hline $\begin{array}{l}\text { O programa é capaz de apoiar a decisão sobre as prioridades de remediação entre dois } \\
\text { ou mais lixões? }\end{array}$ & 5 & 4 & 5 & 5 & 4,8 \\
\hline $\begin{array}{l}\text { Tendo em vista que o programa classifica os cenários de remediação para determinado } \\
\text { lixão em ordem decrescente de pontuação e que o cenário de maior pontuação é o mais } \\
\text { indicado, a ordem dos cenários estabelecida pelo programa está na sequência que você } \\
\text { entende como adequada? }\end{array}$ & 3 & 5 & 5 & 5 & 4,5 \\
\hline $\begin{array}{l}\text { O cenário indicado pelo programa equivale ao do cenário real ou ao cenário que você } \\
\text { entende como adequado? }\end{array}$ & 2 & 5 & 5 & 5 & 4,3 \\
\hline $\begin{array}{l}\text { Quão próximos dos motivos reais estão os motivos fornecidos pelo programa para a } \\
\text { indicação do cenário (destacado em amarelo no rodapé da aba cenário)? }\end{array}$ & 3 & 4 & 5 & 5 & 4,3 \\
\hline $\begin{array}{l}\text { As técnicas de remediação indicadas pelo programa são equivalentes às técnicas } \\
\text { aplicadas no caso real ou as que você entende como adequadas para a remediação } \\
\text { do lixão? }\end{array}$ & 4 & 5 & 5 & 5 & 4,8 \\
\hline \multicolumn{5}{|c|}{ Média Geral } & 4,6 \\
\hline
\end{tabular}

Legenda: $\mathrm{E} 1$ = Especialista 1; E2 = Especialista 2; E3 = Especialista 3; E4 = Especialista 4 
Portanto, o programa desenvolvido atende à proposta deste artigo, sobretudo para a realidade brasileira, onde a maior parte dos municípios é de pequeno a médio porte e suas prefeituras não possuem corpo técnico qualificado para o atendimento à PNRS no que diz respeito à remediação e à eliminação dos lixões. Além disso, o ReLix também demonstrou ser capaz de auxiliar a remediação de lixões de RSU em nível mundial, desde que seja implementada a tradução para outros idiomas.

\section{CONCLUSÕES}

O ReLix foi projetado para atender às necessidades de países que precisam remediar e eliminar seus lixões, como no caso do Brasil e da República de Cabo Verde, onde grande parte dos municípios é de pequeno a médio porte e não possui corpo técnico especializado em remediação de lixões.

Nesse sentido, o programa possibilita fazer diagnóstico de lixões de RSU por meio de um sistema de pontuação, o qual estabelece o nível de impacto do lixão, classifica os cenários de remediação indicando o cenário mais propenso e sugere as técnicas de remediação a serem utilizadas.

Para validar o ReLix, o mesmo foi aplicado em dez lixões no Brasil e em Cabo Verde por uma banca de quatro especialistas, que o avaliaram sendo como capaz de fornecer um diagnóstico apropriado a lixões de RSU, estabelecendo o nível de impacto e indicando as técnicas e cenário de remediação com $92 \%$ de confiabilidade, permitindo que as partes interessadas façam uma análise caso a caso para garantir tomadas de decisão apropriadas sobre as prioridades de remediação e a seleção do cenário de remediação de seus lixões.

\section{CONTRIBUIÇÃO DOS AUTORES}

Todos os autores contribuíram de forma igualitária.

\section{REFERÊNCIAS}

ABRELPE. Panorama dos Resíduos Sólidos no Brasil. São Paulo: 2013

ABRELPE. Panorama dos Resíduos Sólidos no Brasil. São Paulo: 2015

ABRELPE. Panorama dos Resíduos Sólidos no Brasil. São Paulo: 2017.

ADEME. Remise en état des décharges : méthodes et techniques. ADEME, Agence de l'Environnement et de la Maîtrise de l'Energie, , 2005. Disponível em: <http://www.ademe.fr/remise-etat-decharges-methodes-techniques>. Acesso em: 9 maio. 2016.

BARNHART, B. L. et al. Embedding co-production and addressing uncertainty in watershed modeling decision-support tools: Successes and challenges. Environmental Modelling \& Software, v. 109, p. 368-379, 1 nov. 2018. https://doi. org/10.1016/j.envsoft.2018.08.025

BEZERRA, E. Princípios de Análise e Projeto de Sistemas Com UML. [s.l: s.n.].

BOOCH, G.; RUMBAUGH, J.; JACOBSON, I. UML: Guia do usuário 2. ed. Rio de Janeiro: [s.n.]

BOSMANS, A. et al. The crucial role of Waste-to-Energy technologies in enhanced landfill mining: a technology review. Journal of Cleaner Production, v. 55, p. 10-23, 15 set. 2013. https://doi. org/10.1016/J.JCLEPRO.2012.05.032

BOYER, I. et al. Decision Tool For Landfill Remediation. Brooks AFB, TX 78235-5363: [s.n.].

BURLAKOVS, J. et al. Paradigms on landfill mining: From dump site scavenging to ecosystem services revitalization. Resources, Conservation and Recycling, v. 123, p. 73-84, 1 ago. 2017. https://doi.org/10.1016/J.RESCONREC.2016.07.007

CASTILHOS JUNIOR, A. B. DE et al. Catadores de materiais recicláveis: análise das condições de trabalho e infraestrutura operacional no Sul, Sudeste e Nordeste do Brasil. Ciência \& Saúde Coletiva, v. 18, n. 11, p. 3115-3124, nov. 2013. https://doi. org/10.1590/S1413-81232013001100002

DALMORO, M.; VIEIRA, K. M. Dilemas na construção de escalas Tipo Likert: o número de itens e a disposição influenciam nos resultados? [s.l.] [Argos Editora Universitária], 2014. v. 6

DUBEY, A.; CHAKRABARTI, M.; PANDIT, D. Landfill Mining as a Remediation Technique for Open Dumpsites in India. Procedia Environmental Sciences, v. 35, p. 319-327, 1 jan. 2016. https:// doi.org/10.1016/J.PROENV.2016.07.012

FEAM. Reabilitação de áreas degradadas por resíduos sólidos urbanos. Belo HorizonteFEAM, , 2010. Disponível em: <http:// www.feam.br/images/stories/Flavia/areas_degradadas.pdf> 
FOWLER, M. UML essencial: Um breve guia para a linguagem padrão de modelagem de objetos. 3. ed. Porto Alegre: [s.n.].

GABBAR, H. A.; ABOUGHALY, M.; AYOUB, N. Comparative study of MSW heat treatment processes and electricity generation. Journal of the Energy Institute, 8 maio 2017. https://doi. org/10.1016/J.JOEl.2017.04.009

GUEDES, G. T. A. UML 2: Uma abordagem prática. 2. ed. São Paulo: [s.n.].

HOORNWEG, D.; BHADA-TATA, P. What a Waste : A Global Review of Solid Waste Management. 1 mar. 2012.

JOSEPH, K. et al. Dumpsite Rehabilitation Manual. Chennai, Chennai-600 025, India: Anna University Chennai, 2008.

MAHESHI, D.; STEVEN, V. P.; KAREL, V. A. Environmental and economic assessment of "open waste dump" mining in Sri Lanka. Resources, Conservation and Recycling, v. 102, p. 67-79, set. 2015. https://doi.org/10.1016/j.resconrec.2015.07.004

MARZOUGUI, A.; BEN MAMMOU, A. Impacts of the dumping site on the environment: Case of the Henchir El Yahoudia Site, Tunis, Tunisia. Comptes Rendus - Geoscience, v. 338, n. 16, p. 1176 1183, dez. 2006. https://doi.org/10.1016/j.crte.2006.09.020

MASI, S. et al. Assessment of the possible reuse of MSW coming from landfill mining of old open dumpsites. Waste management (New York, N.Y.), v. 34, n. 3, p. 702-10, mar. 2014. https://doi. org/10.1016/J.WASMAN.2013.12.013

MONTEIRO, J. H. P. et al. Manual de Gerenciamento Integrado de resíduos sólidos. Rio de Janeiro: [s.n.].

RAMOS, N. F. Proposição de metodologia para apoio à decisão para a recuperação de área degradada por disposição irregu- lar de resíduos sólidos urbanos. [s.l.] Universidade Federal de Santa Catarina, 2016.

RAMOS, N. F. et al. Desenvolvimento de ferramenta para diagnóstico ambiental de lixões de resíduos sólidos urbanos no Brasil. Engenharia Sanitária e Ambiental, v. 22, n. 6, p. 1233-1241, dez. 2017. https://doi.org/10.1590/s1413-41522017165817

SÁNCHEZ, L. E. Avaliação de impacto ambiental: conceitos e métodos. São Paulo: [s.n.].

USEPA. Handbook Remedial Action at Waste Disposal Sites (Revised). Disponível em: <http://nepis.epa.gov/Exe/ZyPURL. cgi?Dockey=2000GVXJ.txt>.

USEPA. LANDFILL MANUALS: LANDFILL OPERATIONAL PRAC-

TICES. Ardcavan, Wexford, Ireland: [s.n.].

USEPA. Technical Guidance For RCRA/CERCLA Final Covers. Washington DC: [s.n.].

USEPA. Fact Sheet on Evapotranspiration Cover Systems for Waste Containment. [s.l: s.n.].

ZAÏRI, M. et al. Rehabilitation of El Yahoudia dumping site, Tunisia. Waste management (New York, N.Y.), v. 24, n. 10, p. 102334, jan. 2004. https://doi.org/10.1016/j.wasman.2004.07.002

ZAMAN, A. U. Comparative study of municipal solid waste treatment technologies using life cycle assessment method. International Journal of Environmental Science \& Technology, v. 7, n. 2, p. 225-234, 1 mar. 2010. https://doi.org/10.1007/ BF03326132

ZHOU, C. et al. A cost-benefit analysis of landfill mining and material recycling in China. Waste Management, v. 35, p. 191-198, 1 jan. 2015. https://doi.org/10.1016/J.WASMAN.2014.09.029 